\title{
Ictus no Comunicable en un Adulto Joven Con Parálisis Cerebral Portador Del Genotipo MTHFR Variante C677T Homocigótica
}

\author{
Noncommunicable Stroke In A Young Adult With Cerebral Palsy Carrying \\ The Homozygous Variant MTHFR C677T Genotype
}

Francisco Javier Ros-Forteza, MD, PhD

\section{Resumen}

La parálisis cerebral (PC) es un grupo de trastornos que afectan el movimiento y el tono muscular o la postura. Es causada por un daño que se produce en el cerebro inmaduro a medida que se desarrolla, con mayor frecuencia antes del nacimiento. Aunque existen pocos estudios en la literatura, se ha visto que el riesgo de ictus está incrementado en este tipo de pacientes. Se presenta el caso de un joven adulto con ictus reciente y portador de PC. Fue encontrado el genotipo MTHFR variante C677T homocigótica, disminución de ácido fólico y vitamina D. El ictus en contexto de PC es un reto para el clínico. Por consiguiente, pensamos que se debe contemplar la posibilidad de ictus en todo paciente con PC, sugiriendo medidas de prevención primaria en todo paciente con PC.

Palabras clave: Ictus no comunicable, Índice de masa corporal, Genotipo MTHFR variante C677T homocigótica, Parálisis cerebral.

\section{Abstract}

Cerebral palsy (CP) is a group of disorders that affect movement and muscle tone or posture. It is caused by damage that occurs in the immature brain as it develops, most often before birth.

Although there are few studies in the literature, it has been seen that the risk of stroke is increased in this type of patients. We present the case of a young adult with a recent stroke and CP carrying. The homozygous variant MTHFR C677T genotype, decreased folic acid and vitamin D were found. Stroke in CP context is a challenge for the clinician. Therefore, we think that the possibility of stroke should be considered in every patient with CP, suggesting primary prevention measures in every patient with CP.

Keywords: Body mass index, Cerebral palsy, Homozygous variant MTHFR C677T genotype, Noncommunicable stroke.

Rev. Ecuat. Neurol. Vol. 29, $\mathrm{N}^{\circ} 2,2020$

\section{Introducción}

La parálisis cerebral (PC) es un grupo de trastornos que afectan el movimiento y el tono muscular o la postura. Es causada por un daño que se produce en el cerebro inmaduro a medida que se desarrolla, con mayor frecuencia antes del nacimiento. Aunque existen pocos estudios en la literatura, se ha visto que el riesgo de ictus está incrementado en este tipo de pacientes. Se presenta el caso de un joven adulto con ictus reciente y portador de PC.

\section{Caso clínico}

Describimos el caso de un varón de 45 años que notó dificultades en bajar escaleras, acudiendo al servicio de urgencias 48 horas después. Sin convulsión, fiebre o traumatismo previos.

Antecedentes personales: siniestro; portador de $\mathrm{PC}$ forma hemiparética-espástica de predomínio derecha sin dispositivo auxiliar de marcha, GMFCS² (Gross Motor Function Classification System) nivel II. Escoliosis major con giba dorsal derecha y epilepsia bien controlada con levetiracetam $500 \mathrm{mg}$ 2 veces por día, carbamazepina $400 \mathrm{mg} 2$ veces por día, topiramato $50 \mathrm{mg} 2$ veces por día e zonisamida $100 \mathrm{mg}$ por día.

A la admisión: peso $69 \mathrm{Kg}$, altura $1.60 \mathrm{~cm}$, IMC 26.9; tensión arterial 164/64 $\mathrm{mmHg}$, frecuencia cardíaca (FC) 56 lpm. ACP: normal. Saturación arterial de oxígeno 96\% y glucosa $99 \mathrm{mg} / \mathrm{dl}$.
Serviço de Neurologia, Unidade Local de Saúde da Guarda, E.P.E., Guarda (Portugal) Departamento de Ciências Médicas, Faculdade de Ciências da Saúde (Universidade da Beira Interior), Covilhã (Portugal)
Correspondencia:

Avenida Rainha D. Amélia, 6301-857 Guarda-Portugal.

Tel: 00351-271200200

Fax 00351-271200305

E-mail: javierros40@hotmail.com 
La exploración neurológica reveló una disartria, paresia facial central derecha y hemiparesia derecha. Fuerza muscular: extremidad superior derecha con secuelas, grado 3 proximal y grado 1 distal; extremidad inferior derecha, grado 4 proximal, grado 3 distal. Reflejos osteomusculares aumentados y reflejo cutáneo plantar em extensión a la derecha. Sin otros halllazgos en la exploración neurológica y sistémica. $\mathrm{Al}$ interrogatorio dirigido, el paciente confirmó el empeoramiento de la fuerza muscular principalmente en la pierna derecha.

Estudio analítico con alteraciones: genotipo MTHFR variante C677T homocigótica; ácido fólico 2.6 $\mathrm{ng} / \mathrm{mL}(3.1-20.05)$ y vitamina $\mathrm{D} 10 \mathrm{ng} / \mathrm{mL}$ (a partir de $30 \mathrm{ng} / \mathrm{mL}$ suficiente).

Restante estudio sin alteraciones: hemograma, proteína $\mathrm{C}$ reactiva, velocidad de sedimentación globular, iones, función renal y hepática, ácido úrico, vitamina B12, perfil lipídico y tiroideo; anticuerpos anti Treponema pallidum, HIV-1 y 2; autoinmunidad; homocisteína, factor V Leyden, gen de la protrombina G20210A, proteína $\mathrm{C}$, proteína $\mathrm{S}$ y antitrombina III.

ECG: ritmo sinusal (RS), FC 60 lpm

La tomografia computarizada (TC) craneal mostró alteración en la conformación de los lóbulos frontal e insular izquierdos y del núcleo basal izquierdo con ectasia de LCR y calcificaciones cerebrales inespecíficas.
Además alteración de la conformación del seno cavernoso y carótida cavernosa a la izquierda, no pudiéndose excluir la presencia de aneurisma. Atrofia cerebelosa derecha. En la angioTC no se observó flujo en la región de la arteria carótida interna (ACI) izquierda. Presencia de aneurisma antiguo trombosado y calcificado $(2.5 \times 1.5$ $\mathrm{x} 1.5 \mathrm{~cm}$ ). Lesión cerebral extensa hemisférica izquierda para caracterizar por RM.

Fue solicitado el informe de la RM craneal antigua (realizada hace 24 años) sugestiva de hemiatrofia cerebral izquierda incipiente con atrofia del cuerpo calloso y cerebelosa de predominio derecho, lesiones periventriculares izquierdas y aneurisma del sifón carotídeo izquierdo.

Cinco días después del inicio de los síntomas realizó una RM craneal (Figura 1): se confirma una alteración morfo-estrutural hemisférica cerebral izquierda, de predominio lobar frontal izquierda y también del tálamo izquierdo y de los ganglios de la base izquierdos. Se identifica un aumento del espesor del cortex cerebral frontal izquierdo (que es discretamente hiperintenso en T2), se asocia una alteración de la emisión normal de señal de la substancia blanca subcortical frontal y temporal izquierda y peri-ventricular, de la cápsula externa izquierda y de la cápsula interna izquierda, identificando franca hipo-señal en T1 e hiper-señal en T2. Estas alteraciones no tienen efecto de masa, confirmando una atrofia hemisférica
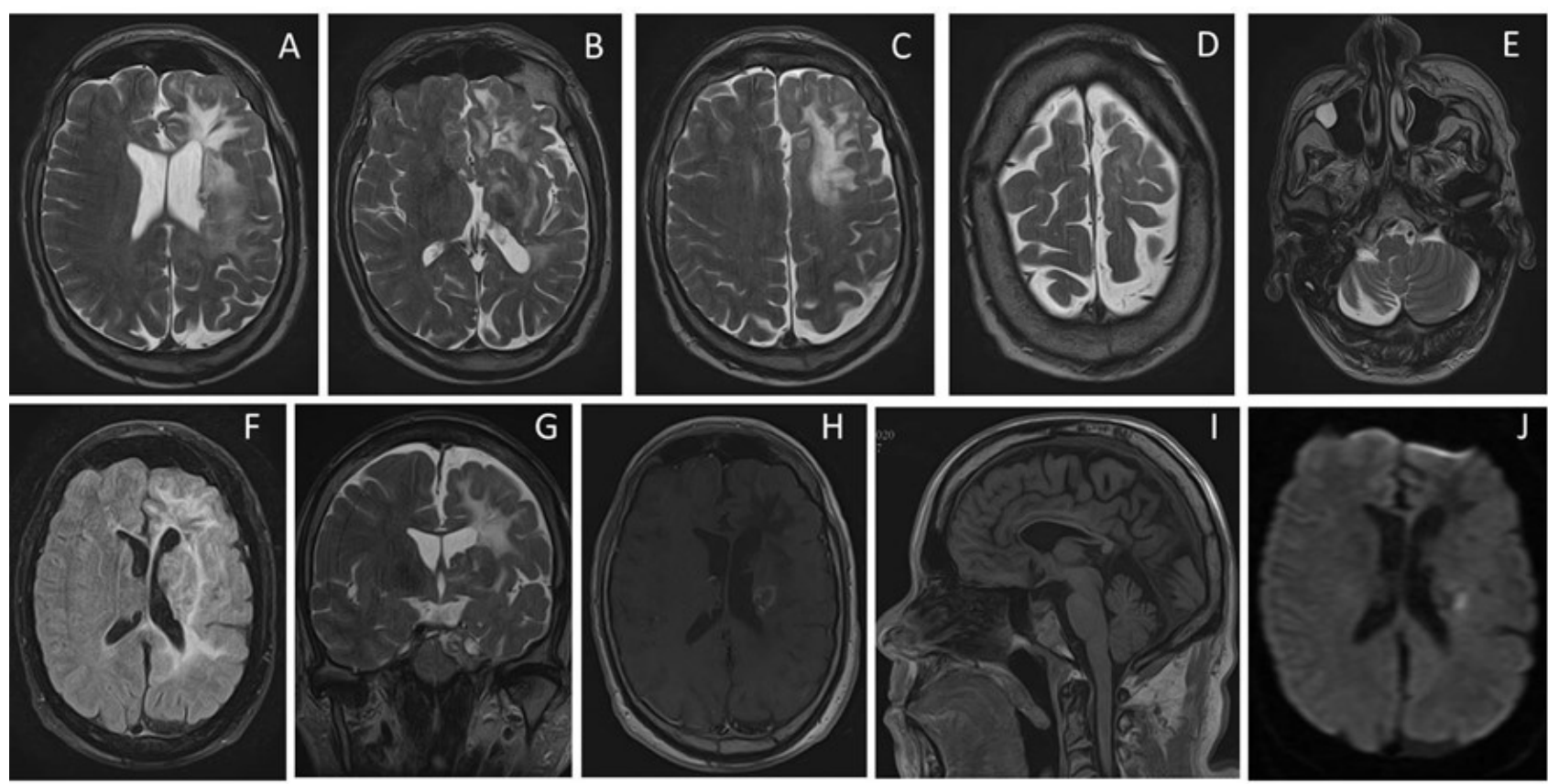

Figura 1. RM craneal: T2 axial (A-E), FLAIR axial (F), T2 coronal (G), T1 axial con gadolinio (H), T1 sagital (I), Difusión axial (J). Alteración morfo-estrutural hemisférica cerebral izquierda, de predomínio lobar frontal izquierda y también del tálamo izquierdo y de los ganglios de la base izquierdos. Aumento del espesor del cortex cerebral frontal izquierdo (que es discretamente hiperintenso en T2), se asocia una alteração de la emisión normal de señal de la substancia blanca subcortical frontal y temporal izquierda y peri-ventricular, de la cápsula externa izquierda y de la cápsula interna izquierda, identificando franca hipo-señal en T1 e hiper-señal en T2. Atrofia hemisférica cerebral izquierda y del pedúnculo cerebral izquierdo. El cuerpo calloso es delgado, con una aparente disgenesia de la rodilla (I). Asimetría de los hemisferios cerebelosos con hipoplasia del hemisferio cerebeloso derecho (E). Alteración de la emisión normal de señal del segmento cavernoso de la $\mathrm{ACl}$ izquierda, correspondiente a aneurisma trombosado (G). Pequeño foco de restricción a la difusión en la corona radiata izquierda (J), que confirma lesión vascular isquémica reciente con realce de contraste a la periferia $(\mathrm{H})$ y con extensión periventricular. 
cerebral izquierda y del pedúnculo cerebral izquierdo. El cuerpo calloso es delgado, con una aparente disgenesia de la rodilla. Se observa una asimetría de los hemisferios cerebelosos con hipoplasia del hemisferio cerebeloso derecho. Adicionalmente, se detecta un pequeño foco de restricción a la difusión en la corona radiata izquierda, que, atendiendo a la clínica, confirma una lesión vascular isquémica reciente con realce de contraste a la perifería y con extensión periventricular. La alteración de la emisión normal de señal del segmento cavernoso de la ACI izquierda, corresponde a um aneurisma trombosado antiguo.

\section{Otras pruebas realizadas}

Ecocardiograma transtorácico: ventrículo izquierdo con hipertrofia del septo basal y fracción de eyección del $77 \%$. Sin masas ni trombos. Ecocardiograma transesofágico: sin shunt, trombo, aneurisma o vegetación.

Holter de 24 h: RS. FC entre 31-86 lpm, raras extrasístoles ventriculares, sin pausas.

Doppler carotídeo-vertebral (Figura 2): ligero aumento difuso del espesor de la íntima-media a lo largo de los ejes carotídeos común e interno. Reducción del calibre y trombosis total de la ACI, condicionando alteraciones espectrales a nivel de la AC común homolateral, la cual muestra un flujo de alta resistencia, con ausencia de diástole. Arteria vertebral izquierda de calibre pericentimétrico, con sentido de flujo fisiológico.

El paciente descrito fue medicado con ácido acetilsalicílico $100 \mathrm{mg}$, rosuvastatina $10 \mathrm{mg}$, ácido fólico $5 \mathrm{mg}$, colecalciferol 800 UI y dieta hipocalórica. Realizó fisioterapia intensiva con evolución favorable. A las 3 semanas reinició su actividad habitual en su institución.

\section{Discusión}

Nuestro paciente era portador de una PC estable, fue detectado un IMC (índice de masa corporal) aumentado sugestivo de pre-obesidad y su epilepsia además estaba bien controlada. Aunque la PC no es una condición progresiva, sin embargo, al menos una cuarta parte de los adultos jóvenes informan que su capacidad para caminar empeora en algún momento de su enfermedad. ${ }^{3}$ Por otra parte, se ha visto que el ictus puede ser una enfermedad no comunicable (ENC) entre los adultos con $\mathrm{PC}, 3$ lo que pudo ocurrir en nuestro caso. Sabemos que existe un incremento del riesgo de ictus en los adultos con PC comparado con los adultos sin PC, aunque los mecanismos subyacentes no están dilucidados. Según Peterson MD et al., 2015, los adultos con PC tienen 5.5 veces más probabilidades de sufrir un ictus. ${ }^{4}$

Aunque la prevalencia de tabaquismo, alcoholismo y obesidad fue más baja en los adultos con PC comparado con los adultos sin PC, sin embargo, es posible que el IMC como métrica carezca de la sensibilidad para detectar altos niveles de grasa corporal porque las personas con PC tienen una menor proporción de masa corporal magra. ${ }^{4}$ Por lo tanto, el IMC puede ser un indicador deficiente en el screening de los factores de riesgo metabólico, como la hiperlipidemia en adultos con $\mathrm{PC}^{5,6}$ y el outcome cardiovascular ser deficiente en personas con PC con puntos de corte de IMC más bajos que los aplicados a la población general. Otros factores potenciales que impulsan la asociación entre la PC y el aumento de la tasa de ENC pueden incluir la inactividad física y el manejo inadecuado de los factores de riesgo de ENC como la hiperlipidemia. ${ }^{3}$ Los estudios de poblaciones sin discapacidad que
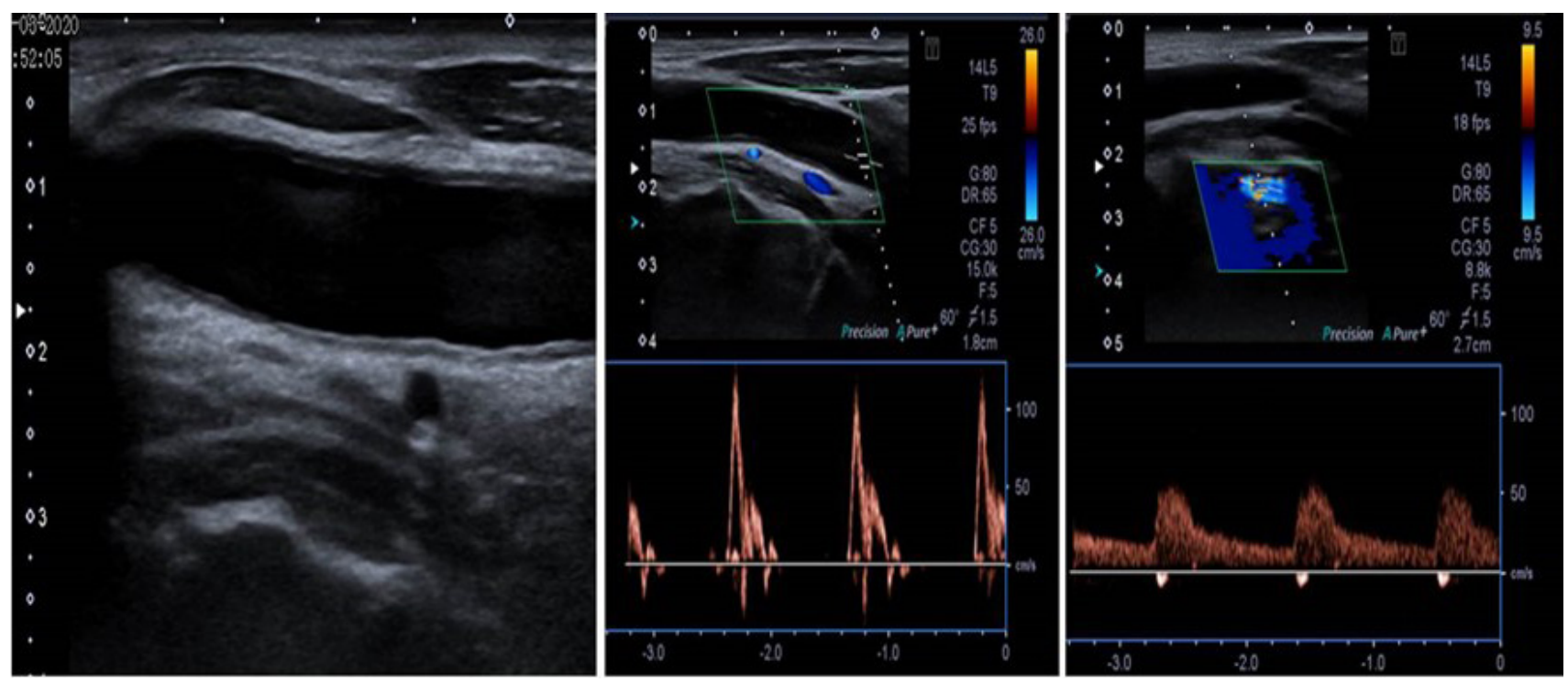

Figura 2. Doppler carotídeo-vertebral: Ligero aumento difuso del espesor de la íntima-media a lo largo de los ejes carotídeos común e interno. Reducción del calibre y trombosis total de la ACl izquierda, condicionando alteraciones espectrales a nivel de la AC común homolateral, la cual muestra un flujo de alta resistencia, con ausencia de diástole. Arteria vertebral izquierda de calibre pericentimétrico, con sentido de flujo fisiológico. 
documentan una asociación de niveles más bajos de actividad física con un mayor riesgo de ictus proporcionan una evidencia adicional de la posible conexión PC-ictus?

A su vez, el déficit de vitamina $D$ es además el nexo con entidades mórbidas relacionadas con la obesidad, tales como síndrome metabólico, enfermedad coronaria, hiperlipidemia, y diabetes mellitus, ${ }^{8}$ y muy probablemente en nuestro caso con el ictus.

De este modo, los adultos de mediana edad con PC tienen alta prevalencia de multimorbilidad, y tanto la obesidad como los niveles más altos de GMFCS se asocian de forma independiente con un mayor riesgo?

Por otra parte, en el estudio de Loes C.A. RuttenJacobs et al., 2016, la mutación MTHFR variante C677T fue asociada con infarto lacunar y enfermedad de pequeño vaso ${ }^{10}$ como sucedió en este caso.

\section{Conclusión}

Nuestro paciente, a pesar de no acudir al hospital desde su empeoramiento funcional, fue diagnosticado y tratado a tiempo con buena evolución, deduciendo que el ictus en contexto de PC es un reto para el clínico. Por consiguiente, pensamos que se debe contemplar la posibilidad de ictus en todo paciente con PC, sugiriendo medidas de prevención primaria en todo paciente con PC.

\section{Referencias}

1. Wu CW, Huang SW, Lin JW, Liou TH, Chou LC, Lin HW. Risk of stroke among patients with cerebral palsy: a population-based cohort study. Dev Med Child Neurol 2017;59:52-56.

2. GMFCS (C) Palisano R, Rosenbaum P, Walter S, Russell D, Wood E, Galuppi B. CanChild Centre for Childhood Disability Research, McMaster University. Dev Med Child Neurol 1997;39:214-223.
3. Ryan JM, Peterson MD, Matthews A, Ryan N, Smith KJ, O'Connell NE, Liverani S, Anokye N, Victor C, Allen E. Noncommunicable disease among adults with cerebral palsy. A matched cohort study. Neurology 2019;93:1385-1396.

4. Peterson MD, Ryan JM, Hurvitz EA, Mahmoudi E. Chronic conditions in adults with cerebral palsy. JAMA 2015;314:2303-2305.

5. Ryan JM, Crowley VE, Hensey O, McGahey A, Gormley J. Waist circumference provides an indication of numerous cardiometabolic risk factors in adults with cerebral palsy. Arch Phys Med Rehabil 2014;95:1540-1546.

6. Peterson MD, Haapala HJ, Hurvitz EA. Predictors of cardiometabolic risk among adults with cerebral palsy. Arch Phys Med Rehabil 2012;93:816-821.

7. Lee CD, Folsom AR, Blair SN. Physical activity and stroke risk: a meta-analysis. Stroke 2003; 34:2475-81.

8. de la Torre-Olivares R, Moreno-Lorenzo C, PérezMármol JM, Cabrera-Martos I, Villaverde-Gutierrez C, Castro Sánchez AM, Aguilar-Ferrándiz ME. Evaluation of functional status associated with overweight in adults with cerebral palsy. Reabilitation Nursing 2016; 0:1-9.

9. Cremer N, Hurvitz EA, Peterson MD. Multimorbidity in Middle-Aged Adults with Cerebral Palsy. Am J Med 2017;130: 744.e9-744.e15.

10. Rutten-Jacobs Loes C.A., Traylor M, Adib-Samii P, Thij V, Sudlow C, Rothwell PM et al. Association of MTHFR C677T Genotype With Ischemic Stroke Is Confined To Cerebral Small Vessel Disease Subtype. Stroke 2016;47:646-651.

Conflictos de interés: El autor declara no tener ningún conflicto de intereses. 